\title{
Simultaneous measurement of the spectral and temporal properties of a LINAC pulse from outside the treatment room
}

\author{
Victor Sanchez-Tembleque, Daniel Sanchez-Parcerisa*, Victor Valladolid-Onecha, Luis Mario Fraile, \\ Jose Manuel Udias
}

Grupo de Física Nuclear y UPARCOS, Departamento de Estructura de la Materia, Física Térmica y

Electrónica, Universidad Complutense de Madrid, CEI Moncloa, 28040 Madrid, Spain.

Instituto de Investigación Sanitaria del Hospital Clínico San Carlos (IdISSC), Madrid, Spain.

*Corresponding author: dsparcerisa@ucm.es

\begin{abstract}
A detection system composed of a $\mathrm{CeBr}_{3}$ scintillating crystal coupled with a photomultiplier tube and connected to a digital oscilloscope was installed in the control room of a Cyberknife ${ }^{\mathrm{TM}}$ clinical accelerator during normal irradiation in order to infer the temporal characteristics of the beam and spectral characteristics of the low-intensity scattered radiation present outside the treatment room.

Three batches of 20,000 pulses were measured at three different gantry angles. After digitalization and post-processing of the signals, the time profile of the beam pulses was determined with an accuracy of $14 \mathrm{~ns}$ with respect to the gating signal, while the energy spectrum of the measured radiation field could be acquired with a resolution of $5.5 \%$.

The proposed device makes it possible, thanks to the increased sensitivity of the scintillating crystals and the digital signal processing techniques in place, to monitor the properties of the radiation present outside the bunker produced by the treatment beam, minimizing interference with patient treatment, QA or research activities.
\end{abstract}

\section{Introduction}

The objective of this work is the development of beam analysis system that can register, from outside the treatment bunker and with high accuracy, the time profile of the gamma rays emitted by a clinical linear accelerator (LINAC). This system is based on digital signal processing of the pulses measured by a fast scintillating crystal with spectroscopic capabilities coupled to a photomultiplier tube (PMT). The system, placed in the control room of the treatment unit, is sensitive to scattered photons emitted from the treatment head when the beam is on.

Traditionally, water-equivalent (or tissue-equivalent) plastic scintillators coupled with PMTs have been used for beam monitoring of radiotherapy devices (Beddar et al 1992, 1992b), since the time decay of the light emitted is on the order of a few ns, allowing for high count rates and time resolutions of the order of $100 \mathrm{ps}$ However, plastic scintillators will not provide useful information on the energy spectra of the photons detected within the radiotherapy range. Over the last decade, new inorganic scintillating crystals such as $\mathrm{LaBr}_{3}(\mathrm{Ce})$ or $\mathrm{CeBr}_{3}$ with a very fast decay time of 20-30 ns have come into the market, allowing for high count rates and time resolutions also in the order of 100 ps (Fraile et al 2013, Vedia et al 2015). In addition, owing to their very good stopping power, they provide good detection efficiency and their energy resolution for photons has no match.

The need for a precise temporal characterization of clinical beams (Sánchez-Tembleque et al 2017) arose during our research in photoacoustic imaging (Kim et al 2017, Giza et al 2018, Sanchez-Parcerisa et al 2018), where an exact knowledge of the time profile of the dose pulse is essential for reconstructing the three-dimensional dose distribution from detected shock waves. 
A key attribute of the proposed system is its ability to perform measurements on the treatment beam from the control room, outside the treatment bunker, where a very limited amount of detectable radiation is present. This is possible thanks to the increased sensitivity of the scintillating crystals (Brownridge et al 1984, Van Eijk 2002), which also provide precise spectral knowledge of the scattered radiation, and it allows the measurements to be carried out without any interference with patient treatment or research activities. Together with the new scintillator materials, full digitization of the pulses was employed in this work, offering us the maximum flexibility for the analysis and allowing us to obtain clean spectra, free from pulse pile-up in a very simple manner.

\section{Experimental setup}

The detector used in this work was a cylindrical Scionix $\mathrm{CeBr}_{3}$ scintillator (1-inch length, 1-inch diameter) coupled to a fast Hamamatsu PMT model R9779. The PMT was connected to a high voltage of 1000V. The dynode signal from the PMT was digitized with an Agilent DSO 6104A oscilloscope with a bandwidth of 1 $\mathrm{GHz}, 8$-bit vertical resolution and a maximum sampling speed of 4 GSamples/s. We selected a sampling speed of 0.1 GSamples/s and a time acquisition window of $10 \mu \mathrm{s}$. At this digitizing speed, the scope used the additional samples to increase the effective vertical resolution to 11 bits. The oscilloscope was read via USB to a PC.

The measurements were performed at the Cyberknife ${ }^{\mathrm{TM}}$ unit (Accuray Technologies, Sunnyvale CA, USA) at of Hospital Ruber Internacional (Madrid, Spain). The unit is equipped with a 6 MV linear accelerator mounted on a 6 degree-of-freedom robotic arm. A circular brass collimator with an aperture size of $5 \mathrm{~mm}$ at a reference distance of $80 \mathrm{~cm}$ was used to shape the field. The dose rate was kept at $600 \mathrm{MU} / \mathrm{min}$. In order to study geometrical and scattering effects, measurements were taken with the robotic arm in horizontal, vertical and oblique $\left(45^{\circ}\right)$ positions, and no target. Furthermore, to investigate in-patient scattering, an additional measurement was taken with the arm in vertical position and the beam irradiating a cubic $(1 \mathrm{~m}$ side) water phantom, positioned at a source-surface distance of $48 \mathrm{~cm}$. The detector system was placed in the Cyberknife ${ }^{\mathrm{TM}}$ control room (outside the shielding maze), at an approximate distance of $5 \mathrm{~m}$ from the isocenter and behind a $1.8 \mathrm{~m}$-wide wall of concrete (see Figure 1).

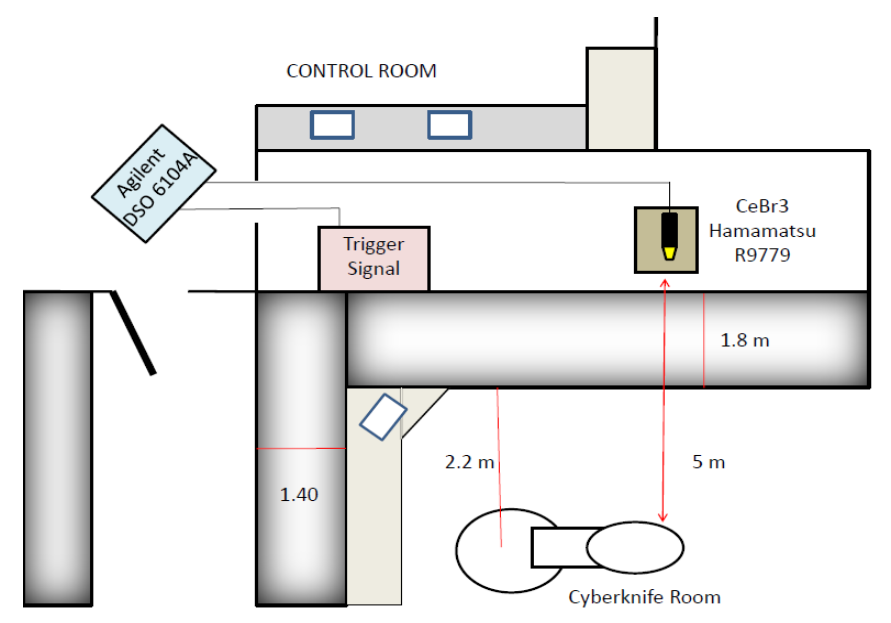

Figure 1. Sketch of the treatment room showing the relative position of the detection system and the treatment unit. 
The acquisition was triggered using the magnetron output signal (MAG) from the Cyberknife ${ }^{\mathrm{TM}}$ control unit. This signal is active whenever the high voltage magnets are energized, regardless of whether the beam is on or not, which allowed us to use the same trigger signal for the background measurements. This signal is approximately square, with a duration of about $5 \mu \mathrm{s}$ and a pulse repetition frequency of $188 \mathrm{~Hz}$.

\section{Digital signal processing}

Every time the trigger signal goes active, the oscilloscope captures $10 \mu$ s from the dynode output of the PMT. A batch of signals then consists of several captures (20,000 samples per measurement), which must then be post-processed to obtain the time profile and energy spectrum of the beam.

\subsection{Background subtraction}

Radiotherapy treatment rooms are environments with high electromagnetic noise, due to the presence of radiofrequency fields and high-voltage switching devices. This noise can easily alter the measurements of sensitive electronics. Moreover, the possible presence of activated radioactive elements can contribute to an increased background radiation being detected by the scintillating crystal. It is therefore necessary to perform a background measurement and to use it to correct all subsequent acquisitions.

Before the start of irradiation, we took a background measurement of 2,000 samples with the high voltage magnets energized and the robotic arm in vertical position. Due to the low duty cycle of our measurements (below $0.1 \%$ ), the rate of random events (radiation captured at the scintillating crystal but not originating from the beam) is extremely low in comparison with the signal from scattered radiation present outside the treatment room during irradiation. The main contribution to the background is the electromagnetic noise caused by the accelerator, which appears synchronized with the trigger signal and is present regardless of whether the beam is on or off. Figure 2 displays a superposition of 15 consecutive traces (in red), together with the noise synchronous background signal (in black) and the trigger signal (in blue). It is not possible to avoid this synchronous noise by averaging consecutive signals, and it must therefore be subtracted from all measured pulses in the post-processing stage.

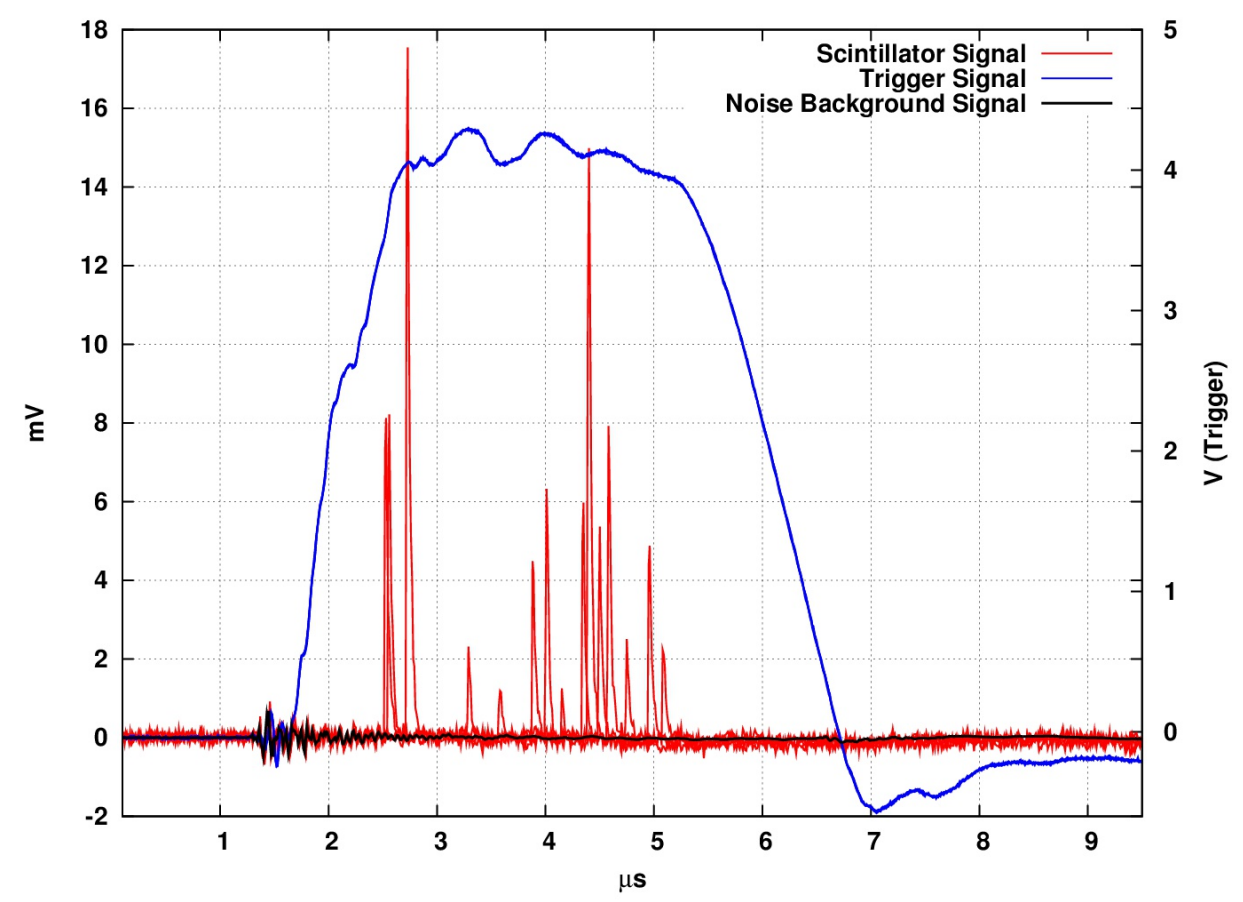

Figure 2. In red, superposition of 15 signals measured by the $\mathrm{CeBr}_{3}-$ PMT detector containing one photon each. In black, background signal averaged from 2,000 samples. All signals are synchronized with the MAG trigger (blue line). 


\subsection{Pile-up events}

The high voltage gain of the PMT coupled to the $\mathrm{CeBr}_{3}$ crystal and the trigger level in the scope are adjusted to count individual photons arriving at the detector. Because of the high pulse rate in the detector when the trigger is on, different photon events can be captured in the same time window in a single trigger cycle (what we call time-window pile-up events). This effect can alter the derivation of temporal and spectral profiles from the set of collected signals and must thus be corrected for.

The correction for pile-up events was based on a shape-factor study of the pulse. There are different ways to relate pulse shape with incoming photon energy, with the simplest of them using either the maximum pulse value or the pulse integral (obtained via trapezoid rule). Therefore, we expect a linear correlation between these two values, as they both are expected to have a linear correlation with the incoming photon energy. When more than one photon hits the detector during a single acquisition window, the correlation between maximum pulse height and pulse integral is changed, and we can use this to filter out these pile-up events.

For our measurements, we applied a cut-off to events where the integral value does not follow a linear correlation with the maximum pulse value to filter out pile-up events, setting the threshold (solid line in Figure 3) at $20 \%$ of the median of the integral values for a given maximum value. Events reaching the maximum dynamic range of the digitizer were also filtered out. The ratio of filtered events ranged from $2.5 \%$ of the pulses (in vertical position) to 7\% of the pulses (in horizontal position, where the event rate is highest). The distortion introduced in the spectral measurements by this filtering is minimal.

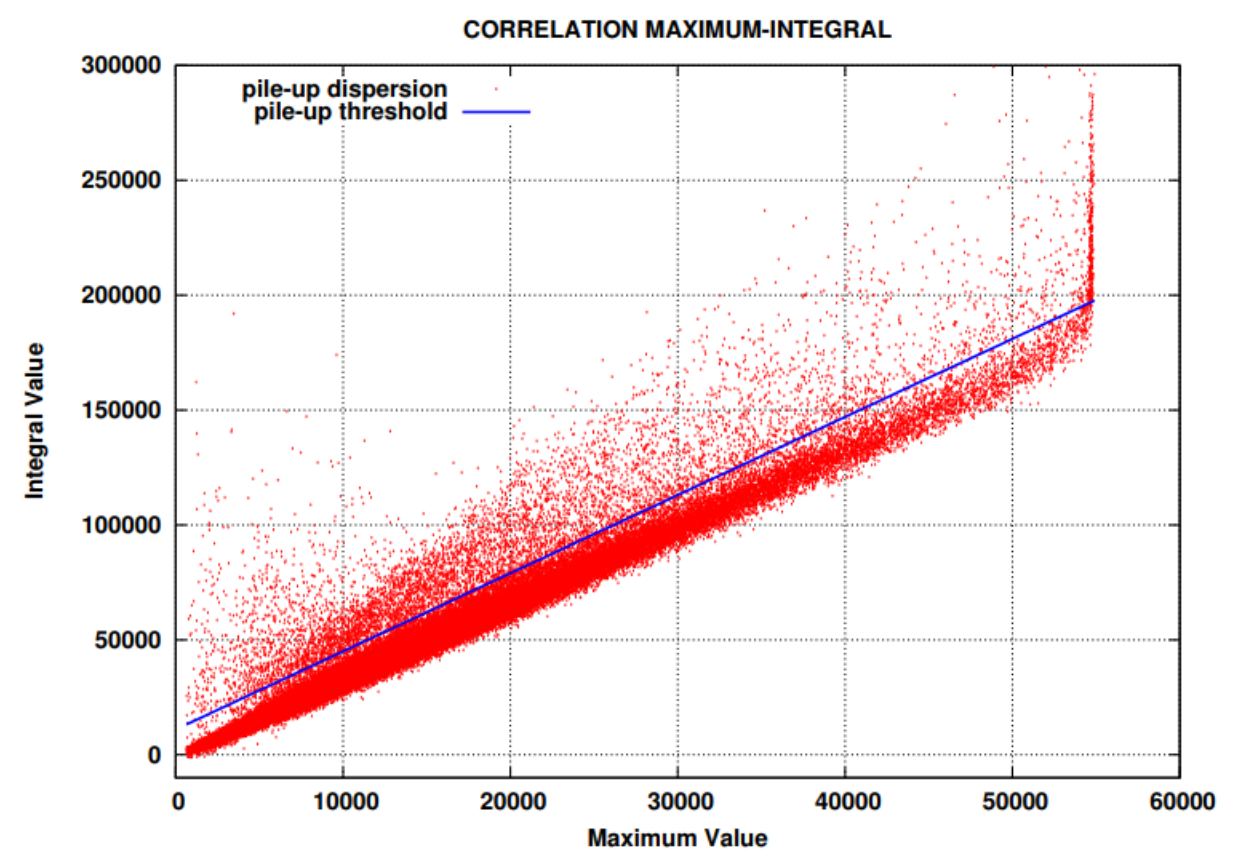

Figure 3. Pulse integral value (in arbitrary units) vs. pulse maximum value for 20,000 events measured with the robotic arm in horizontal position. Line marks the threshold above which events are considered pile-up and discarded.

\subsection{Derivation of time profiles}

Time profiles are extracted from the series of individual photons (such as the ones shown in Figure 2). For each photon (excluding pile-up events), the pulse arrival time is determined from its leading edge, detected when the signal crosses a threshold of $0.5 \mathrm{mV}$ and stays above that threshold for more than 3 consecutive samples $(30 \mathrm{~ns})$. The accuracy of this algorithm is determined by the sampling time of our system, which amounts to $14 \mathrm{~ns}$ with respect to the trigger signal. 


\subsection{Derivation of spectral information of the scattered radiation in the control room}

The energy of the pulses is determined from the integral of the detected pulse. Once a valid pulse has been captured (duration over $30 \mathrm{~ns}$ ), the system calculates the definite integral using the trapezoidal method between the position of the maximum value and a position delayed $100 \mathrm{~ns}$ with respect to it. The correlation between pulse integral values and photon energy values was determined using the $511-\mathrm{keV}$ and $1175-\mathrm{keV}$ full-energy peaks of a ${ }^{22} \mathrm{Na}$ calibration source, acquired in self-trigger mode with high voltage magnets in the room turned off. The FWHM energy resolution of the system in these calibration peaks was estimated at $5.5 \%$ at $511 \mathrm{keV}$.

As we have mentioned in section 3.2, pile-up events were excluded from the spectral analysis. The obtained spectra were also corrected for the efficiency of the detector (shown in Figure 4), calculated via Monte Carlo simulation with penEasy/PENELOPE (Salvat et al 2008, Picado et al 2017) under the assumption that the incidence angle of photons is roughly perpendicular to the base of the scintillating crystal and taking into account that the dimensions of the crystal are similar in all axis (1-inch in length and diameter), thus minimizing the influence of the angle of incidence in the efficiency to less than $3 \%$ for incidences up to $45^{\circ}$ (and less than $10 \%$ for perpendicular incidences, significantly less likely to occur), according to our simulations.

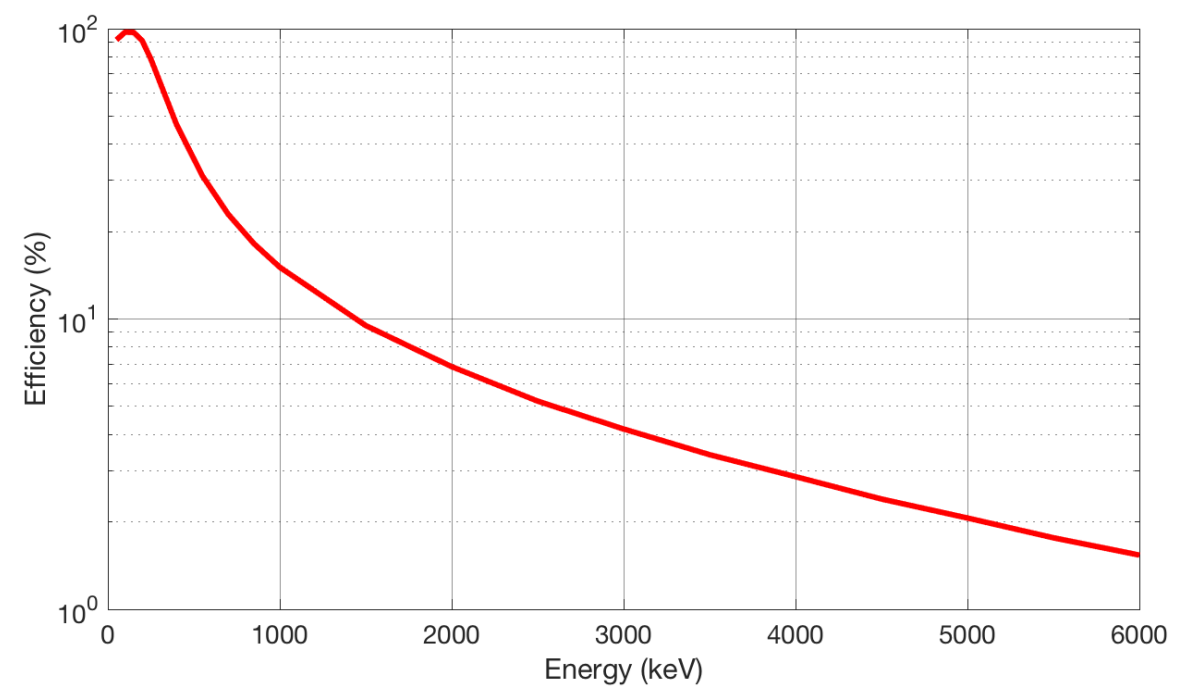

\section{Figure 4. Detector efficiency as a function of the energy of the incoming photons,} calculated via Monte Carlo simulation.

\section{Results}

In the reported irradiation tests, our $\mathrm{CeBr}_{3}$-PMT detection system registered with 100-ns accuracy the time profiles for different beam orientations. Each of the profiles shown in Figure 5 corresponds to an average of 20,000 trigger events, post-processed for background subtraction and pile-up filtering. Three acquisitions were performed without a target at three different gantry angles (horizontal, vertical and 45 degrees), plus a fourth one, in vertical orientation, using a water phantom as a target. The four measured profiles show a quasi-identical time behavior and differ only in the number of events. As we expect from the distribution of Compton events, when the beam is placed horizontally pointing towards the detector, the number of registered events increases, and it is minimum when the beam is pointing downwards. There are no significant differences between the profiles obtained with the beam in vertical position with and without a water target, from where we can deduce that most measured photons originate in the treatment nozzle and the contribution of particles scattered from the target is minimal.

The energy spectra measured in the control room for the three different orientations are displayed in Figure 6. These energy spectra registered at the measurement position (see Figure 1), after potentially several Compton scattering events and significant hardening of the beam through the shielding material and modulated by the energy response from the scintillating crystal (Figure 4). At low energies (below $600 \mathrm{keV}$ ), 
the energy distribution of received photons seems to be independent of the position of the accelerator (within uncertainty ranges). However, above this threshold, we observe a higher proportion of energetic photons for more direct beam incidence positions. This is expected, as photons arriving to the detector in less direct trajectories have undergone more Compton scattering and thus have a lower energy. The average photon energies are $0.69 \mathrm{MeV}, 0.67 \mathrm{MeV}$ and $0.62 \mathrm{MeV}$ for the horizontal, oblique and vertical positions, respectively.
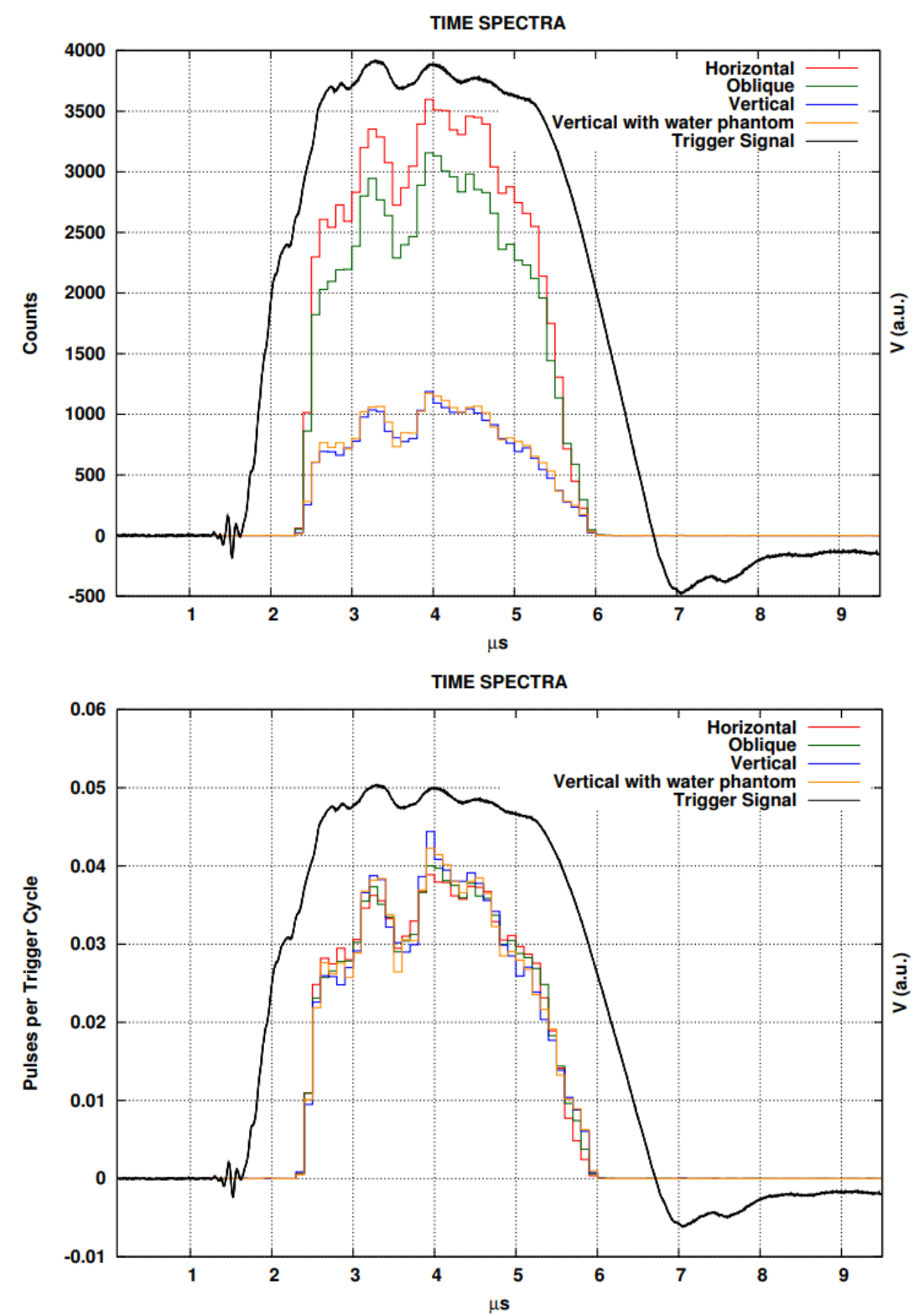

Figure 5. Top: Time fluence profiles registered for the four different beam configurations (horizontal, oblique, vertical, vertical with target) for $\mathbf{2 0 , 0 0 0}$ triggered events. Bottom: Time fluence profiles normalized to the number of captured events (i.e. non-empty trigger cycles). 

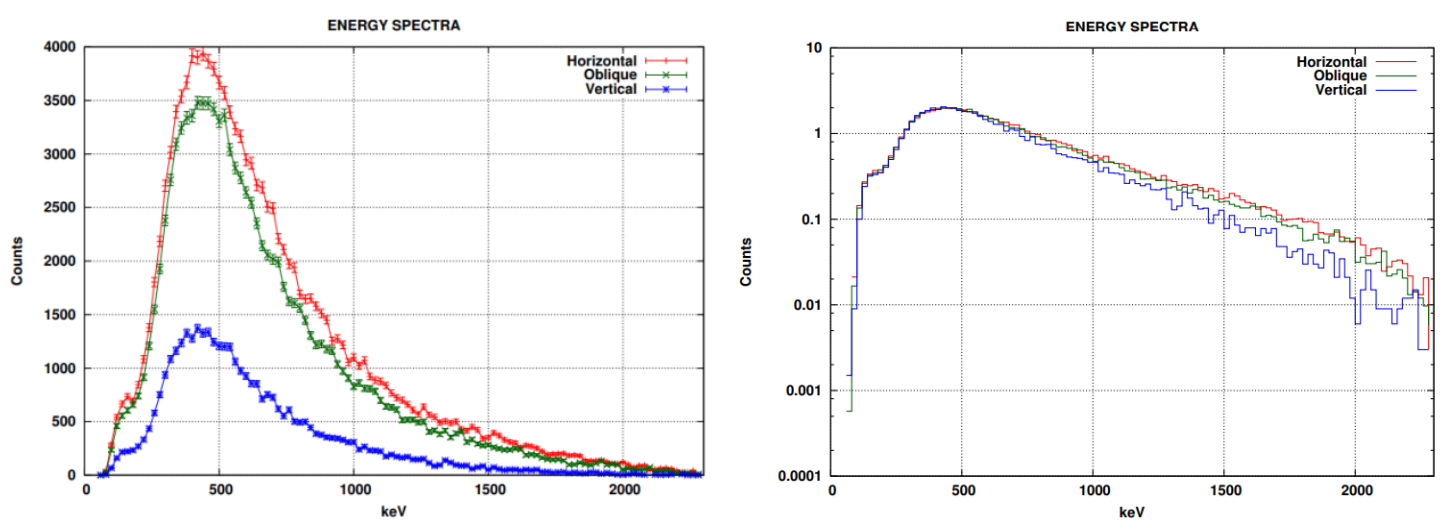

Figure 6. Measured energy spectra for different beam orientations and without target, displayed in linear (left) and log (right) scales, corrected for detector response.

\section{Discussion and conclusions}

With the presented system, it is possible to acquire simultaneously, from the LINAC control room, the time profile of the dose pulse and the energy spectrum of the scattered radiation outside the bunker. This characterization of the beam is relevant not only for research purposes (gammaacoustics, development of new detectors) but it could constitute a useful tool to assess the performance and stability of the LINAC. For instance, an increase in the ratio of radiation-empty pulses (or a decrease in the average number of photons detected per pulse) could be an indication of incorrect machine operation.

Fully digital treatment of the pulses allows us to easily filter the intense electromagnetic noise and simplifies the derivation of the energy spectrum of the received photons, maximizing the information obtained from the detector without the need for additional complex electronics.

Batch measurements of 20,000 triggered signals, at a pulse rate repetition of $188 \mathrm{~Hz}$, were acquired in less than 2 minutes. Even placing our detectors outside the treatment room, we are able to capture, on average, more than one usable (i.e. not piled-up) photon per trigger at all beam orientations $(1.3,3.7$ and 4.3 photons per trigger for the vertical, oblique and horizontal angles, respectively), with the fraction of triggered pulses without any photon acquisition at $24.7 \%, 1.1 \%$ and $0.4 \%$ at the three respective angles (Figure 7 ). This rate is sufficient to acquire enough statistics during normal patient, QA or research irradiation. Further analysis of multi-photon events could, if needed, increase the number of usable events and therefore the acquisition efficiency. Moreover, an analysis of the proportion of empty pulses and mean number of photons per pulse could be an indication of the correct operation and stability of the LINAC, since a higher-than-usual number of radiation-empty pulses could be linked with system malfunction. 

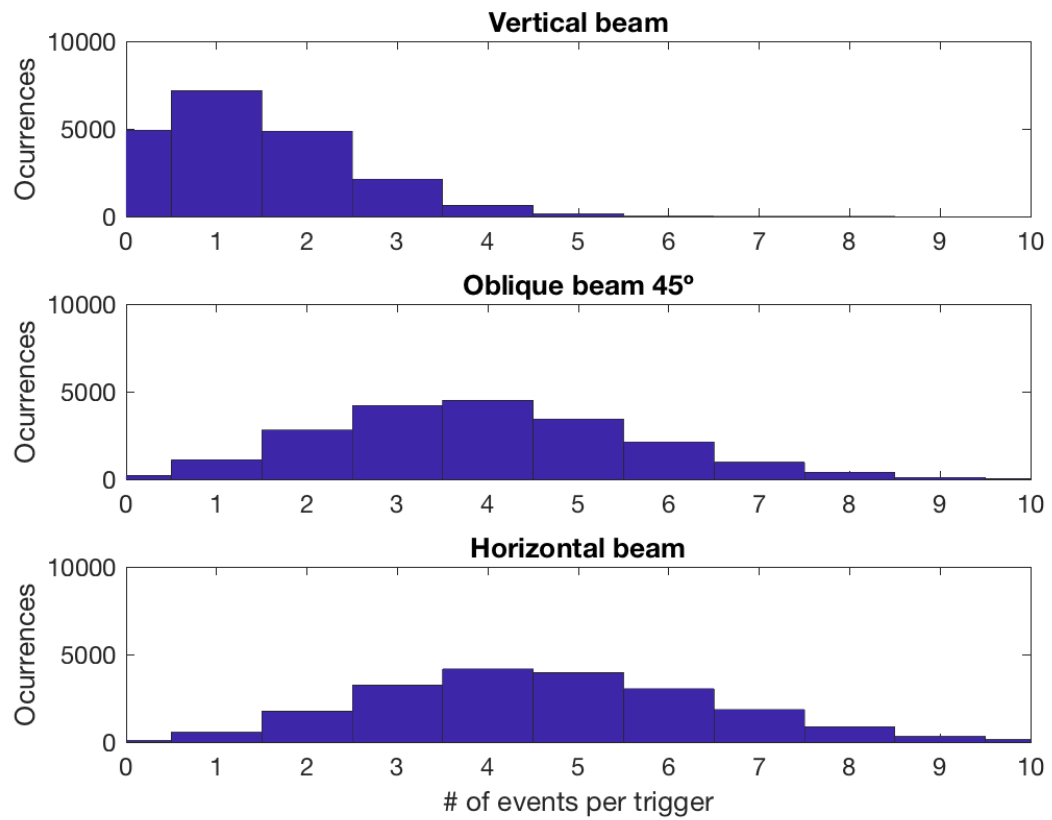

Figure 7. Number of events per trigger at the three different beam orientations.

The energy spectra measured outside the bunker (Figure 6) cannot be used to derive full spectral information from the treatment beam. Even with a full Monte Carlo analysis (Ma et al 1999, Ding 2002, Verhaegen \& Seuntjens 2003) including simulation of the treatment nozzle and room shielding geometry and spectrum correction for angular-dependent detector efficiency (Picado et al, 2017), the derived spectra are still limited by the response of the crystals to photon energies above $1.5 \mathrm{MeV}$ (Figure 4). Moreover, the pile-up correction negatively affects the registration of energetic photons (above $2 \mathrm{MeV}$ ).

In summary, the proposed setup has been successfully used to accurately measure the time profile and energy spectrum of the scattered radiation outside the treatment room from a Cyberknife ${ }^{\mathrm{TM}}$ clinical accelerator. 


\section{Acknowledgements}

Work supported by the Spanish Government (FPA2015-65035-P, RTC-2015-3772-1), Comunidad de Madrid (S2013/MIT-3024 TOPUS-CM) and European Regional Funds. This is a contribution for the Moncloa Campus of International Excellence, "Grupo de Física Nuclear-UCM", Ref. 910059. The authors acknowledge partial support by EU's H2020 under MediNet: a Networking Activity of ENSAR-2 (grant agreement 654002). The authors want to thank the service of Medical Physics of Hospital Ruber Internacional, especially Francisco Fayos and Elena Antolín, for their support and assistance during the experiments.

\section{References}

1. Beddar, A.S., Mackie, T.R. and Attix, F.H., 1992. Water-equivalent plastic scintillation detectors for highenergy beam dosimetry: I. Physical characteristics and theoretical considerations. Physics in medicine and biology, 37(10), p.1883.

2. Beddar, A.S., Mackie, T.R. and Attix, F.H., 1992b. Water-equivalent plastic scintillation detectors for highenergy beam dosimetry: II. Properties and measurements. Physics in medicine and biology, 37(10), p.1901.

3. Brownridge, J., Samnick, S., Stiles, P., Tipton, P., Veselka, J. and Yeh, N., 1984. Determination of the photon spectrum of a clinical accelerator. Medical physics, 11(6), pp.794-796.

4. Ding, G.X., 2002. Energy spectra, angular spread, fluence profiles and dose distributions of 6 and 18 MV photon beams: results of Monte Carlo simulations for a Varian 2100EX accelerator. Physics in medicine and biology, 47(7), p.1025.

5. Fraile, L.M., Mach, H., Vedia, V., Olaizola, B., Paziy, V., Picado, E. \& Udias, J.M., 2013. Fast timing study of a $\mathrm{CeBr}_{3}$ crystal: Time resolution below 120 ps at ${ }^{60} \mathrm{Co}$ energies. Nucl. Instrum. Methods A 701, pp. 235-242.

6. Giza O., Sanchez-Parcerisa D., Sanchez-Tembleque V., Herraiz J.L., Camacho J., Avery S. \& Udias J.M., 2018. Photoacoustic dose monitoring in clinical high-energy photon beams. Phys. Med. Biol, submitted.

7. Hinson, W.H., Kearns, W.T., Deguzman, A.F. and Bourland, J.D., 2008. Photon spectral characteristics of dissimilar 6MV linear accelerators. Medical Physics 35(5), pp.1698-1702.

8. Kim J, Park EY, Jung Y, Kim BC, Kim JH, Yi CY, Kim IJ, Kim C, 2017. X-Ray Acoustic-Based Dosimetry Using a Focused Ultrasound Transducer and a Medical Linear Accelerator. IEEE Transactions on Radiation and Plasma Medical Sciences 6, pp. 534-40.

9. Ma, C.M. and Jiang, S.B., 1999. Monte Carlo modelling of electron beams from medical accelerators. Physics in medicine and biology, 44(12), p. R157.

10. Malka, V., Faure, J. and Gauduel, Y.A., 2010. Ultra-short electron beams based spatio-temporal radiation biology and radiotherapy. Mutation Research/Reviews in Mutation Research, 704(1), pp.142-151.

11. Picado, E., Carmona-Gallardo, M.; Cal-González, J.; Fraile, L.M.; Mach, H.; Udías, J.M. \& Vedia, V, 2017. Efficiency measurement and Monte Carlo simulations of a CeBr3. Applied Rad. Isotopes 120, pp. 71-75.

12. Salvat, F., Fernández-Varea, J. M., \& Sempau, J. (2008, June). PENELOPE-2008: A code system for Monte Carlo simulation of electron and photon transport. In the Workshop Proceedings, June.

13. Sanchez-Tembleque V., Sanchez-Parcerisa D., Fraile L.M., Udias J., 2017. Medida simultánea del espectro y el perfil temporal del pulso de un LÍNAC desde fuera de la sala. XXXVI Meeting of the Spanish Physics Society, Symposium on Nuclear Physics, Santiago, $21^{\text {st }}$ July 2017.

14. Sanchez-Parcerisa, D., Giza, O., Camacho, J., Sanchez-Tembleque, V., Avery, S., \& Udias, J, 2018. EP-1734: Photo-and proto-acoustic dose monitoring in radiosurgery and proton beams. Radiotherapy and Oncology, 127 , p. S928.

15. Van Eijk, C.W., 2002. Inorganic scintillators in medical imaging. Physics in medicine and biology 47(8), p. R85.

16. Vedia, V., Mach, H., Fraile L.M., Udías, J.M. \& Lalkovski, S., 2015. Enhanced time response of 1-in. $\mathrm{LaBr}_{3}(\mathrm{Ce})$ crystals by leading edge and constant fraction techniques. Nucl. Instrum. Methods A 795, pp. 24450 .

17. Verhaegen, F. and Seuntjens, J., 2003. Monte Carlo modelling of external radiotherapy photon beams. Physics in medicine and biology, 48(21), p. R107. 